mary outcome that is specified in the protocol is critical in clinical trials, and any changes during the conduct of the trial must precede data unblinding and be well justified and well documented. In the present case, the authors followed these standards, but by changing the primary outcome to a less specific one, they may have inadvertently reduced the probability of observing a true difference in event rates between the trial groups.

Thus, although the investigators concluded that antibiotic prophylaxis before miscarriage surgery "did not result in a significantly lower risk of pelvic infection, as defined by pragmatic broad criteria, than placebo" I would interpret the results as indicating that antibiotic prophylaxis prevented pelvic infections as defined by international diagnostic criteria. Given the risks associated with pelvic infections in LMICs, these data provide reasonable support for prescribing prophylactic antibiotics in these settings. Antibiotic resistance, however, will need to be monitored.
Disclosure forms provided by the author are available with the full text of this editorial at NEJM.org.

From the Makerere University School of Public Health, Kampala, Uganda.

1. Dellicour S, Aol G, Ouma P, et al. Weekly miscarriage rates in a community-based prospective cohort study in rural western Kenya. BMJ Open 2016;6(4):e011088.

2. D'Ubaldo C, Pezzotti P, Rezza G, Branca M, Ippolito G. Association between HIV-1 infection and miscarriage: a retrospective study. AIDS 1998;12:1087-93.

3. Mellerup N, Sфrensen BL, Kuriigamba GK, Rudnicki M. Management of abortion complications at a rural hospital in Uganda: a quality assessment by a partially completed criterionbased audit. BMC Womens Health 2015;15:76-85.

4. Fawcus S, McIntyre J, Jewkes RK, et al. Management of incomplete abortions at South Africa public hospitals. S Afr Med J 1997;87:438-42.

5. Lissauer D, Wilson A, Hewitt CA, et al. A randomized trial of prophylactic antibiotics for miscarriage surgery. N Engl J Med 2019;380:1012-21

6. Abortion in Africa: incidence and trends. Washington, DC: Guttmacher Institute, March 2018 (https://www.guttmacher.org/ fact-sheet/abortion-Africa).

7. Brown HC, Jewkes R, Levin J, Dickson-Tetteh $\mathrm{K}$, Rees $\mathrm{H}$. Management of incomplete abortion in South African public hospitals. BJOG 2003;110:371-7.

DOI: 10.1056/NEJMe1900872

Copyright (๑) 2019 Massachusetts Medical Society.

\title{
Human Genetics and Drug Development
}

\author{
Michael V. Holmes, M.D., Ph.D.
}

Current tools in the field of human genetics can reliably estimate both the intended and the adverse effects of modifying a therapeutic target to guide the clinical development of medicines. ${ }^{1}$ The distinction between an adverse effect of a drug that is target-mediated, and thus a characteristic of all drugs in the same class, and an adverse effect that is off-target, affecting typically an individual drug (rather than a drug class), is critical to decision making in drug development. Although relevant studies of human genetics cannot characterize off-target effects (Fig. 1), extrapolations may be feasible in certain limited circumstances.

For example, in the Investigation of Lipid Level Management to Understand Its Impact in Atherosclerotic Events (ILLUMINATE) trial, the addition of torcetrapib, a cholesteryl ester transfer protein (CETP) inhibitor, led to a higher risk of cardiovascular disease than the control treatment. ${ }^{2}$ In a large meta-analysis of data from individual participants, the presence of CETP variants that mimic the therapeutic inhibition of CETP was associated with a lower risk of cardiovascular disease. ${ }^{3}$ Such qualitative heterogeneity between genetically predicted risk of disease and treatment-elicited risk of disease can signal the presence of off-target effects that affect an individual drug (although alternative explanations exist). ${ }^{4}$ Phenotypic comparisons of a drug with its target-encoding gene can pinpoint the mechanisms of such off-target effects, enabling rapid screening of other drugs in the same class for the presence of similar off-target effects. Thus, the selection of which drugs to take forward into clinical development can be guided by studies in human genetics.

The characterization of the associations of genetic variants with multiple phenotypes (so-called phenomewide association studies, or PheWAS) and with disease end points can elucidate the repertoire of target-mediated effects, potentially motivating genetically informed drug repurposing for new indications. For example, genetic variants mimicking therapeutic inhibition of the interleukin-6 receptor (monoclonal antibody inhibitors are licensed for the treatment of rheumatoid arthritis) provided early support for a causal role of inflammation in coronary heart disease, ${ }^{5}$ find- 
ings that were subsequently validated in the Canakinumab Antiinflammatory Thrombosis Outcome Study (CANTOS). ${ }^{6}$

Ray et al. ${ }^{7}$ now report in the Journal the results of the CLEAR (Cholesterol Lowering via Bempedoic Acid, an ACL-Inhibiting Regimen) Harmony trial, in which 2230 patients with atherosclerotic cardiovascular disease or heterozygous familial hypercholesterolemia (or both) who had a lowdensity lipoprotein (LDL) cholesterol level of at least $70 \mathrm{mg}$ per deciliter (1.8 $\mathrm{mmol}$ per liter) and were taking maximally tolerated statin therapy were randomly assigned in a 2:1 ratio to receive bempedoic acid, an ATP citrate lyase (ACL) inhibitor that acts upstream of 3-hydroxy-3-methylglutaryl coenzyme A (HMG-CoA) reductase (HMGCR) to inhibit cholesterol synthesis, or placebo for 1 year.

The primary end point of safety, assessed by means of the incidence of adverse events and changes in safety laboratory variables during the trial according to the Medical Dictionary for Regulatory Activities, did not differ meaningfully between the bempedoic acid group and the placebo group. However, patients in the bempedoic acid group had an excess risk of adverse events leading to discontinuation of the blinded trial regimen, an excess risk of gout, and higher blood concentrations of uric acid than those in the placebo group. In further analyses of nonprimary and secondary end points, there were additional potentially troubling signals, although the $95 \%$ confidence intervals were wide: $0.9 \%$ of the patients treated with bempedoic acid died, as compared with $0.3 \%$ of those in the placebo group (relative risk, 3.24; $95 \%$ confidence interval [CI], 0.73 to 14.34 ); $0.4 \%$ of the patients in the bempedoic acid group and $0.1 \%$ of those in the placebo group died from adjudicated cardiovascular disease (relative risk, 2.99; $95 \%$ CI, 0.36 to 24.82 ); and $0.6 \%$ and $0.1 \%$, respectively, were hospitalized for heart failure (relative risk, 4.49; 95\% CI, 0.57 to 35.38 ).

Although the findings are potentially alarming, the imprecision that is reflected in the $95 \%$ confidence intervals renders the relative risk values virtually meaningless - but let us take a temporary foray into evidence-averse territories and ask ourselves, "Can these effect estimates be unseen?" A curiosity-driven interpretation leads to additional questions - for example, "Are such effects biologically plausible?" Bempedoic acid lowers the LDL cholesterol level by means of in- hibition of ACL, an enzyme upstream of HMGCR (the target of statins). ${ }^{8}$ If ACL inhibition leads solely to reductions in HMG-CoA, the substrate for HMGCR, then the target-mediated diseaserisk profile (constituting both intended and unintended target-mediated effects) of ACL inhibition ought to mimic that of HMGCR inhibition, making data about HMGCR (among the most well-characterized drug targets) theoretically portable to ACL. A caveat here is that because bempedoic acid is a small-molecule prodrug activated by hepatic enzymes, it may show specificity for hepatic ACL (in contrast to muscle ACL), which could hamper such portability. ${ }^{8}$ Given that ACL is upstream of HMGCR in the cholesterol synthesis pathway, it is, in theory, feasible that a diseaserelated (pleiotropic) pathway might branch off from ACL, proximal to HMGCR, which would also limit portability, but such pleiotropic effects of ACL would be target-mediated and therefore amenable to their reliable characterization by studies in human genetics (Fig. 1). ${ }^{9}$ Alternatively, bempedoic acid may have off-target effects (i.e., effects not mediated through ACL inhibition), which would not be quantifiable in studies of human genetics.

In the accompanying study by Ference et al., ${ }^{10}$ the results of which are also reported in this issue of the Journal, a consistency of cardiovascular disease associations, including a lower risk of fatal coronary heart disease, with variants in ACLY was seen when scaled to the same lowering of the LDL cholesterol level as for variants in HMGCR and PCSK9. These genetic associations for ACLY speak only to target-mediated effects of ACL inhibition, whereas the genetic associations for HMGCR and PCSK9, although representing the target-mediated effects of statin therapy and PCSK9 inhibition, respectively, can also be considered to represent the target-mediated effects of ACL inhibition acting by means of more downstream pathways. In comparing the disease associations of variants in ACLY with variants in HMGCR (Fig. 2 and Table 2 of the article by Ference et al.), the authors are in effect investigating the presence of targetmediated pleiotropy of ACL inhibition, as shown in Fig. 1. Of note, the association of diabetes with ACLY was directionally opposite to that observed with HMGCR (and PCSK9), a finding that was also seen in the CLEAR Harmony trial (relative risk of new-onset or worsening diabetes with bempedoic acid vs. placebo, $0.61 ; 95 \%$ CI, 0.41 to 0.92 ) 


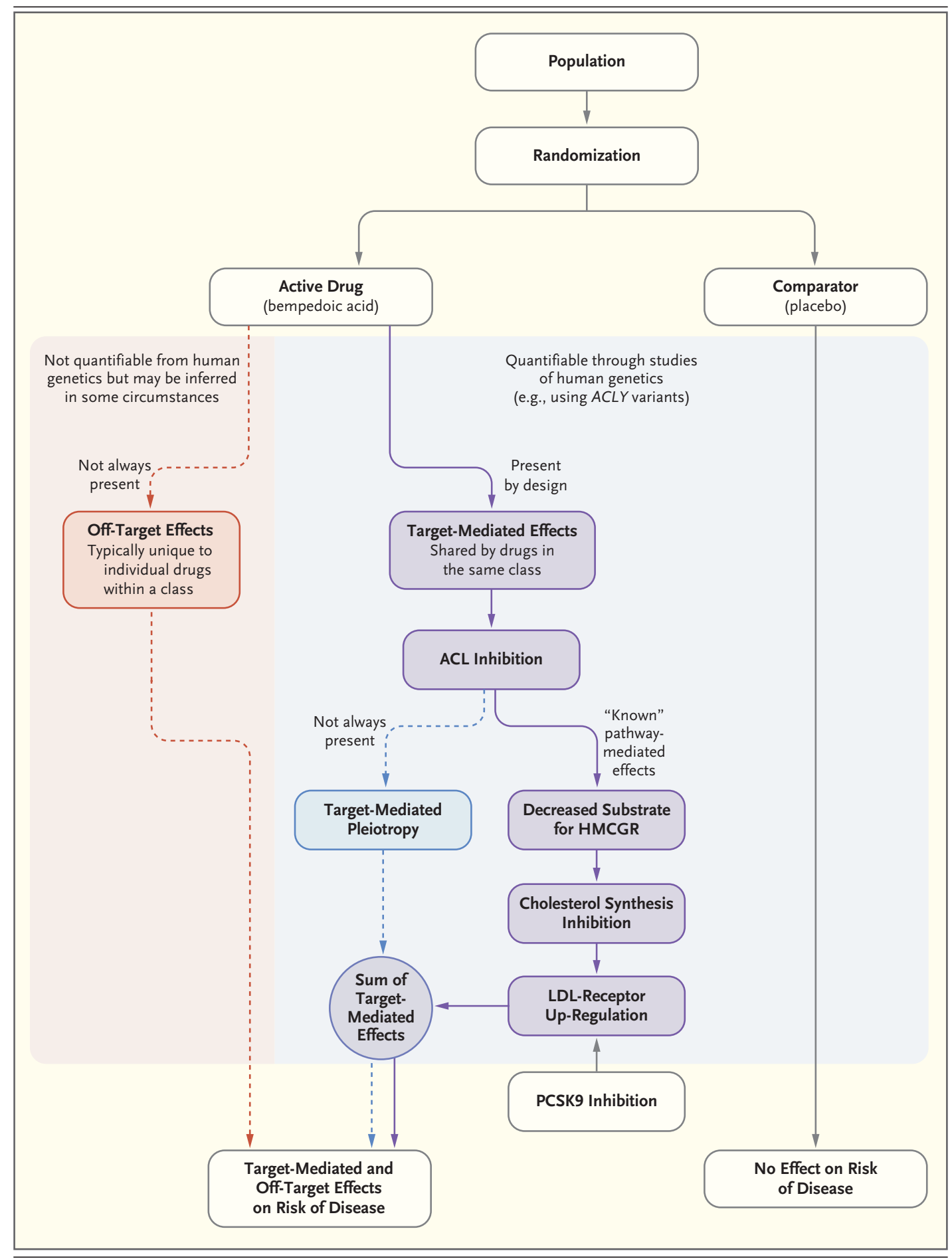

and one that potentially supports the presence of target-mediated pleiotropic effects.

What do the cumulative data from randomized treatment trials and from studies of human given reduction in the LDL cholesterol level, drugs that inhibit ACL (and do not have off-target effects) ought to yield reductions in the risk of cardiogenetics say about ACL inhibition? First, for a achieved with statins. Second, analysis of end 
Figure 1 (facing page). Randomized, Controlled Trials, Human Genetics, and Target-Mediated versus Off-Target Effects.

In this figure, a conventional treatment trial involves randomization to bempedoic acid or placebo. Targetmediated effects of bempedoic acid (in purple) include inhibition of ATP citrate lyase (ACL), with consequential reductions in 3-hydroxy-3-methylglutaryl coenzyme A (HMG-CoA), the substrate for HMG-CoA reductase (HMGCR). This situation leads to inhibition of cholesterol synthesis, up-regulation of hepatic lowdensity lipoprotein (LDL) receptors, and lowering of blood concentrations of LDL cholesterol. A hypothetical target-mediated pleiotropic pathway branching off proximal to HMGCR (e.g., altering the risk of diabetes) is shown in blue. Together, these effects constitute the target-mediated effects of ACL inhibition, which can be reliably quantified by means of studies of naturally occurring variation in the gene that encodes the therapeutic target (e.g., ACLY). In the absence of disease-relevant target-mediated pleiotropy (or tissuespecific effects), existing data from genetic variants in HMGCR and PCSK9 (the gene encoding proprotein convertase subtilisin-kexin type 9) may be portable to $A C L Y$, since they alter LDL cholesterol by means of the same mechanism (i.e., the up-regulation of hepatic LDL receptors). Hypothetical off-target effects cannot be quantified from human genetics, but in some (rare) circumstances, heterogeneity between the drug effects and the corresponding, target-encoding genetic associations can highlight the potential presence of off-target effects, which can be further investigated by means of comparisons of phenotypic associations of the drug with its target-encoding gene to elucidate offtarget mechanisms.

points in treatment trials for which there is marginal statistical power is best avoided; fortunately for bempedoic acid, an ongoing phase 3, cardiovascular outcome trial (CLEAR Outcomes; ClinicalTrials.gov number, NCT02993406) will provide additional information. More broadly, with unprecedented availability of open-access biobanks that are linked to electronic medical records and genomewide genotyping and emerging phenotyping (e.g., proteomics and metabolomics), the genetic characterization of drug targets is set to revolutionize how we develop medicines.

Disclosure forms provided by the author are available with the full text of this editorial at NEJM.org.

From the Medical Research Council Population Health Research Unit and the Clinical Trial Service Unit and Epidemiological Studies Unit, Nuffield Department of Population Health, University of Oxford, Oxford, United Kingdom.

1. Holmes MV, Simon T, Exeter HJ, et al. Secretory phospholipase A(2)-IIA and cardiovascular disease: a mendelian randomization study. J Am Coll Cardiol 2013;62:1966-76.

2. Barter PJ, Caulfield M, Eriksson M, et al. Effects of torcetrapib in patients at high risk for coronary events. N Engl J Med 2007;357:2109-22.

3. Ference BA, Kastelein JJP, Ginsberg HN, et al. Association of genetic variants related to CETP inhibitors and statins with lipoprotein levels and cardiovascular risk. JAMA 2017;318:947-56.

4. Holmes MV, Ala-Korpela M, Smith GD. Mendelian randomization in cardiometabolic disease: challenges in evaluating causality. Nat Rev Cardiol 2017;14:577-90.

5. The Interleukin-6 Receptor Mendelian Randomisation Analysis (IL6R MR) Consortium. The interleukin-6 receptor as a target for prevention of coronary heart disease: a mendelian randomisation analysis. Lancet 2012;379:1214-24.

6. Ridker PM, Everett BM, Thuren T, et al. Antiinflammatory therapy with canakinumab for atherosclerotic disease. N Engl J Med 2017;377:1119-31.

7. Ray KK, Bays HE, Catapano AL, et al. Safety and efficacy of bempedoic acid to reduce LDL cholesterol. N Engl J Med 2019; 380:1022-32.

8. Pinkosky SL, Newton RS, Day EA, et al. Liver-specific ATPcitrate lyase inhibition by bempedoic acid decreases LDL-C and attenuates atherosclerosis. Nat Commun 2016;7:13457.

9. Davies NM, Holmes MV, Davey Smith G. Reading Mendelian randomisation studies: a guide, glossary, and checklist for clinicians. BMJ 2018;362:k601.

10. Ference BA, Ray KK, Catapano AL, et al. Mendelian randomization study of ACLY and cardiovascular disease. N Engl J Med 2019;380:1033-42.

DOI: 10.1056/NEJMe1901565

Copyright @ 2019 Massachusetts Medical Society. 